Proc. XII Int. School on Theoretical Physics - Symmetry and Structural Properties of Condensed Matter

\title{
On Generalized Landau Levels
}

\begin{abstract}
P. KRASOŃ ${ }^{a, *}$ AND J. MILEWSKI ${ }^{b}$
${ }^{a}$ Department of Mathematics and Physics, Szczecin University, Wielkopolska 15, 70-415 Szczecin, Poland

${ }^{b}$ Institute of Mathematics, Poznań University of Technology, Piotrowo 3A, 60-965 Poznań, Poland

We consider the dispersion of energy levels for both standard and inverted quantum harmonic oscillators in the presence of a uniform electromagnetic field. For this analysis we use a solution of the corresponding eigenproblem in terms of the Kummer functions. We find a complete description of the energy levels for a particle of mass $m$ and electric charge $q$ subject to the action of a harmonic oscillator and simultaneous uniform magnetic and electric fields. We also analyze the effect of spin on energy levels for an electron.
\end{abstract}

DOI: 10.12693/APhysPolA.132.94

PACS/topics: 03.65.--w, 03.50.De, 02.30.Gp

\section{Introduction}

In 1927 Landau [1] solved the eigenproblem for a charged particle in a homogeneous magnetic field. The Landau levels and their generalisations are of continuous interest [2-8]. These are useful in analysis of quantum Hall effect [9] and level crossings in graphene [10]. On the other hand, since their introduction quantum harmonic oscillators, both standard and inverted (repulsive) (cf. [11-14]), have proved to be one of the most useful models in particle physics. We analyse the effect of applying a uniform electromagnetic field to the energy levels of a quantum harmonic oscillator. We investigate an eigenproblem for a particle on a plane in a constant homogeneous electromagnetic field along with the potential of a harmonic oscillator. The magnetic field is directed perpendicularly to the plane and the electric field is parallel to the plane. The direction of both potential forces is taken to be parallel to the $x$-axis. The $y$-part of the wave function for a suitable Landau gauge is a plane wave with a wave number $k_{y}$. We consider both standard and inverted one-dimensional harmonic oscillators, acting in the direction of the electric field. In the papers [2] and [3] a particle in electromagnetic field is considered, in [4], [5], and [6] an influence of anisotropic harmonic oscillator is taken into account. Our model corresponds to the limit case of strongly anisotropic oscillator coupled with an electromagnetic field. The direct case was considered in [6], here we deal also with an inverted oscillator. Using the Landau gauge we study the dependence of the dispersion of energy levels on the wave vector contrary to the fact that the standard Landau levels are independent of the wave vector.

\section{Separation of the Hamiltonian}

We consider the Hamiltonian for a particle of mass $m$ with electric charge $q$ on a plane in a uniform magnetic field $\boldsymbol{B}$ in harmonic oscillator potential interacting with

\footnotetext{
*corresponding author; e-mail: piotrkras26@gmail.com
}

homogeneous electric field energy $U$. It is given by the following formula:

$$
\hat{H}=\frac{1}{2 m}(-\mathrm{i} \hbar \nabla-q \boldsymbol{A})^{2}+U
$$

for

$$
U=\frac{1}{2} K x^{2}-q \mathcal{E} x, \boldsymbol{A}=B[0, x]
$$

(the Landau gauge).

The substitution

$$
\Psi(x, y)=\chi(x) \mathrm{e}^{\mathrm{i} k_{y} y}
$$

yields the following equation for $\chi(x)$ :

$$
\hat{H}_{x} \chi(x)=E \chi(x),
$$

where

$$
\hat{H}_{x}=\frac{\hat{p}_{x}^{2}}{2 m}+\frac{1}{2} m \omega_{c}^{2}\left(x-x_{c}\right)^{2}+\frac{1}{2} K x^{2}-q \mathcal{E} x,
$$

$\omega_{c}=\frac{q B}{m}, x_{c}=\frac{p_{y}}{q B}, p_{y}=\hbar k_{y}$. The quantity $\omega_{c}$ is called the cyclotron frequency, $p_{y}$ is an eigenvalue of the momentum operator in the $y$-direction and $x_{c}$ corresponds to the center of the cyclotron orbit for the gauge (2). Let us put

$$
K^{\prime}=m \omega_{c}^{2}+K
$$

For $K, K^{\prime} \neq 0$ we obtain the following form:

$$
\begin{aligned}
\hat{H}_{x} & =\frac{\hat{p}_{x}^{2}}{2 m}+\frac{1}{2} K^{\prime}\left(x-x_{0}\right)^{2} \\
& +\frac{\hbar^{2}}{2 m} \frac{K}{K^{\prime}}\left(k_{y}-k_{0}\right)^{2}-\frac{1}{2 K} q^{2} \mathcal{E}^{2},
\end{aligned}
$$

where $x_{0}=\frac{m \omega_{c}^{2} x_{c}+q \mathcal{E}}{K^{\prime}}, k_{0}=\frac{m \omega_{c}}{\hbar K} q \mathcal{E}$. The eigenproblem for a direct oscillator $(K>0)$, was solved in [6]. In the next section we deal also with the inverted oscillator, which leads us to some new interesting cases. For the particular case $K=0, K^{\prime} \neq 0$ one obtains $[2,15]$ :

$$
\hat{H}_{x}=\frac{\hat{p}_{x}^{2}}{2 m}+\frac{1}{2} K^{\prime}\left(x-x_{0}\right)^{2}-\hbar \frac{\mathcal{E}}{B} k_{y}-\frac{1}{2 K^{\prime}} q^{2} \mathcal{E}^{2} .
$$

The case $m \omega_{c}^{2}+K=0$ yields the same operator as for a particle in a homogeneous potential field

$$
\hat{H}_{x}=\frac{\hat{p}_{x}^{2}}{2 m}-\left(m \omega_{c}^{2} x_{c}+q \mathcal{E}\right) x+\frac{1}{2} m \omega_{c}^{2} x_{c}^{2} .
$$

The eigenproblem for Eq. (9) leads to the Airy equation [16]. 


\section{Dispersion of energy levels}

We recall very shortly some basic facts, needed later, concerning harmonic oscillators both direct and (less known) repulsive. This is done mainly for fixing notation. In one-dimensional case the harmonic oscillator is described by the following Hamiltonian:

$$
\hat{H}=-\frac{\mathrm{i} \hbar^{2}}{2 m} \frac{\mathrm{d}^{2}}{\mathrm{~d} x^{2}}+\frac{1}{2} K x^{2} .
$$

The eigenfunctions can be chosen with defined parity because the potential is an even function. The solution can be given by means of the Kummer function (confluent hypergeometric function of type $(1,1))$ (cf. [16-18]):

$$
\Psi_{p, K, \lambda}(x)=f_{\alpha, p}(x)_{1} F_{1}\left(-\nu+\frac{1}{4}+\frac{p}{2} ; \frac{1}{2}+p ; \alpha x^{2}\right),(11)
$$
where $p=0,1$ and $\lambda=\frac{2 m E}{\hbar^{2}}, \alpha^{2}=\frac{m K}{\hbar^{2}}, \nu=\frac{\lambda}{4 \alpha}$, $f_{\alpha, p}(x)=x^{p} \mathrm{e}^{-\alpha x^{2} / 2}$.

It is very well known that for $K>0$, the spectrum of $\lambda$ and energy is non-degenerate and discrete. For $K<0$ the spectrum is continuous, equal to $\mathbb{R}$ and doubly degenerated [13].

The eigenfunctions of the operator (5) for $K, K^{\prime} \neq 0$ have the following form:

$$
\chi_{p, K, B, \mathcal{E}, \lambda}(x)=\Psi_{p, K^{\prime}, \lambda}\left(x-x_{0}\right),
$$

where $\alpha^{2}=\frac{m K^{\prime}}{\hbar^{2}}$. The law of energy dispersion as a function of $k_{y}$ has the following parabolic form:

$$
\begin{aligned}
& E\left(k_{y}\right)=E\left(k_{0}\right)+D\left(k_{y}\right), \\
& E\left(k_{0}\right)=\frac{\hbar^{2}}{2 m} \lambda-\frac{1}{2 K} q^{2} \mathcal{E}^{2}, \\
& D\left(k_{y}\right)=\frac{\hbar^{2}}{2 m} \frac{K}{K^{\prime}}\left(k_{y}-k_{0}\right)^{2} .
\end{aligned}
$$

For $K^{\prime}>0$, the values of $\lambda$ are non-degenerate and in the discrete set $\lambda_{n}=(1+2 n) \lambda_{0}$, where $\lambda_{0}=\frac{\sqrt{m K^{\prime}}}{\mathrm{i}} \hbar$. Hence

$$
E_{n}\left(k_{0}\right)=\left(\frac{1}{2}+n\right) \hbar \omega^{\prime}-\frac{1}{2 K} q^{2} \mathcal{E}^{2}, \quad \omega^{\prime}=\sqrt{\frac{K^{\prime}}{m}} .
$$

For $K^{\prime}<0$ the values of $\lambda$ are doubly degenerated and fill in the whole real axis.

We see that the shape of energy levels depends on the sign at $\left(k_{y}-k_{0}\right)^{2}$ in Eq. (13):

$K>0$ - levels bent with arms heading upwards, non-degenerated discrete spectrum of $E\left(k_{0}\right)$;

$-m \omega_{c}^{2}<K<0$ - levels bent with arms heading downwards, non-degenerated discrete spectrum of $E\left(k_{0}\right)$;

$K<-m \omega_{c}^{2}-$ levels bent with arms heading upwards, doubly degenerated continuous spectrum of $E\left(k_{0}\right)$.

For $K=0, K^{\prime} \neq 0$ we put $k_{0}:=0$ and from Eq. (8) we obtain a linear function of $k_{y}$ describing the dispersion of energy levels

$$
E_{n}\left(k_{y}\right)=E_{n}\left(k_{0}\right)+D\left(k_{y}\right),
$$

$$
\begin{aligned}
& E_{n}\left(k_{0}\right)=\frac{\hbar^{2}}{2 m} \lambda_{n}-\frac{1}{2 K^{\prime}} q^{2} \mathcal{E}^{2}, \\
& D\left(k_{y}\right)=-\hbar \frac{\mathcal{E}}{B} k_{y} .
\end{aligned}
$$

For $\mathcal{E}=0$ this is the standard case of constant discrete Landau levels.

When $K=-m \omega_{c}^{2}$ cf. Eq. (9) we have a qualitative change of eigenfunctions, they express in generally by means of the Airy function. In Figs. 1-3 we displayed the character of dispersion of Landau levels for various values of $K$.

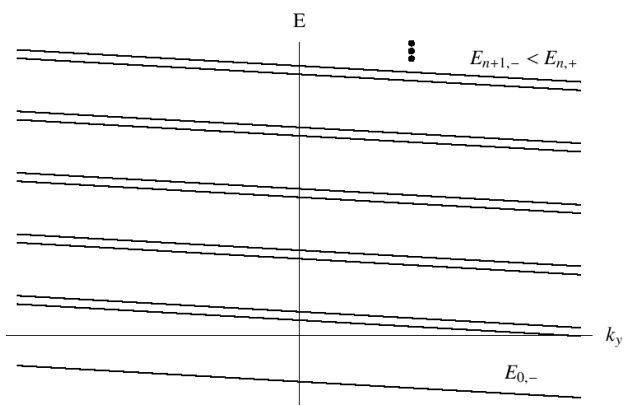

Fig. 1. Dispersion of Landau levels in an electromagnetic field, $K=0$. Non-zero slope is due to the presence of an electric field. Splitting of energy levels is due to the presence of a magnetic field.

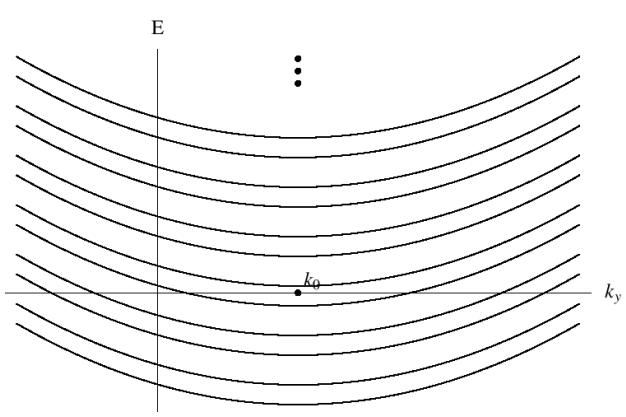

Fig. 2. Qualitative picture for $K>0$ of energy levels for electron in a harmonic oscillator and in a constant electromagnetic field. Note that here we have three possibilities for relative positions of $E_{n,+}$ versus $E_{n+1,-}$.

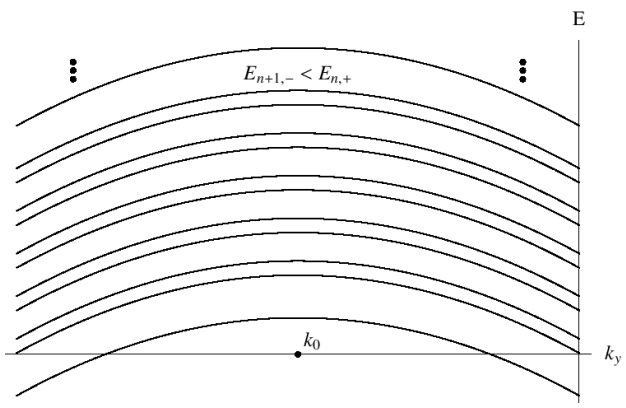

Fig. 3. Qualitative picture for $-m \omega_{c}^{2}<K<0$ of energy levels for electron in a potential of a harmonic oscillator and in a constant electromagnetic field. 


\section{Dispersion of energy levels for an electron}

For a charged particle with spin in an electromagnetic field, the complete Hamiltonian is a sum of the spatial and spin parts

$$
\hat{H}_{\text {tot }}=\hat{H}+\hat{H}_{\text {spin }} \text {. }
$$

An eigenproblem for the spatial part was considered in previous sections. The spin part has the following form:

$$
\hat{H}_{\text {spin }}=-g \frac{\hbar q}{2 m} \boldsymbol{B} \cdot \hat{\boldsymbol{s}},
$$

where $g$ is a gyroscopic factor ( $g$-factor).

The eigenvalues for the spin part are as follows:

$$
E_{\text {spin }}=-\frac{g}{2} \sigma \mathrm{i} \hbar \omega_{c}, \quad \sigma=-s, \ldots, s .
$$

Each of the energy levels splits into $2 s+1$ sublevels

$$
E_{n, \sigma}\left(k_{y}\right)=E_{n, \sigma}\left(k_{0}\right)+D\left(k_{y}\right),
$$

where

$$
E_{n, \sigma}\left(k_{0}\right)=E_{n}\left(k_{0}\right)-\frac{g}{2} \sigma \mathrm{i} \hbar \omega_{c} .
$$

A gap between adjacent levels is equal to

$$
\frac{g}{2} \hbar\left|\omega_{c}\right| \text { for }|q|=e \text {. }
$$

Now, consider energy levels for an electron in electromagnetic field. Since for an electron $s=\frac{1}{2}$, there are exactly two eigenstates of a spin Hamiltonian. Eigenenergies have the following form:

$$
E_{n, \pm}\left(k_{y}\right)=E_{n}\left(k_{y}\right) \pm \frac{g}{4} \mathrm{i} \hbar\left|\omega_{c}\right|,
$$

Based on his equation, Dirac has shown that the $g$ factor for an electron equals $g=2$ [19]. Thus, according to this theory for $K=0$ we have the following degeneration:

$$
E_{n,+}\left(k_{y}\right)=E_{n+1,-}\left(k_{y}\right) .
$$

However, taking into account a correction due to quantum electrodynamics for Dirac's theory the $g$-factor should be taken as $g \approx 2.002319[20,21]$. This means that for $K=0$ we have

$$
E_{n,+}\left(k_{y}\right)>E_{n+1,-}\left(k_{y}\right),
$$

although the gap between these levels is very small. More precisely, the following relations hold:

for $K<K_{g}: E_{n,+}\left(k_{y}\right)>E_{n+1,-}\left(k_{y}\right)$,

for $K=K_{g}: E_{n,+}\left(k_{y}\right)=E_{n+1,-}\left(k_{y}\right)$,

for $K>K_{g}: E_{n,+}\left(k_{y}\right)<E_{n+1,-}\left(k_{y}\right)$,

where $K_{g}=\frac{g^{2}-4}{4} m \omega_{c}^{2}$. Notice that we have obtained two critical values of the spring constant $K: K_{c}=-m \omega_{c}^{2}$, at which there is a change in the spectrum from discrete to continuous, and $K_{g}$, for which the Rabi levels are doubly degenerated. Observe that $K_{c}<0$, which corresponds to a suitable inverted oscillator interacting with the electromagnetic field such that $K^{\prime}=0$.

\section{Conclusions and discussion}

We derived a law for the dispersion of energy versus a wave number $k_{y}$ of a wave function. It is interesting that despite that the direction of potential forces is along $x$-axis the dispersion depends explicitly on the wave number $k_{y}$. It was shown that this law is parabolic for
$K \neq 0$. In the case where $K=0$, the dependence has a linear form i.e. $E\left(k_{y}\right)=a k_{y}+b$. For $K \neq-m \omega_{c}^{2}$ the eigenfunctions can be expressed by means of confluent Kummer functions, whereas for $K=-m \omega_{c}^{2}$ by means of the Airy functions. Moreover, the value $K=-m \omega_{c}^{2}$ can be regarded as the critical one in the sense that above this value the spectrum is discrete and below it is continuous.

\section{Acknowledgments}

The research was partially sponsored by the grant NCN. The second author was partially supported by the grant 04/43 PS PB / 0087 of Ministry of Science and High Education of Poland.

\section{References}

[1] L.D. Landau, Z. Phys. 45, 430 (1927).

[2] G. Konstantinou, K. Maulopoulos, arXiv:1609.00041v1, 2016.

[3] T. Kramer, C. Bracher, M. Kleber, J. Opt. B Quant. Semiclass. Opt. 6, 21 (2004).

[4] T.K. Rebane, Teor. Eksp. Khim. 5, 3 (1969).

[5] T.K. Rebane, Opt. Spectrosc. 112, 813 (2012).

[6] Q.-G. Lin, Commun. Theor. Phys. 38, 667 (2002).

[7] I.D. Vagner, V.M. Gvozdikov, P. Wyder, HIT J. Sci. Eng. A 3, 5 (2006).

[8] Wei Gao-Feng, Long Chao-Yun, Long Zheng-Wen, Qin Shui-Jie, Chin. Phys. C 32, 247 (2008).

[9] T. Taychatanapat, K. Watanabe, T. Taniguchi, P. Jarillo-Herrero, Nat. Phys. 7, 621 (2011).

[10] M. Serbyn, D.A. Abanin, Phys. Rev. B 87, 115422 (2013).

[11] L.D. Landau, E.M. Lifshitz, Quantum Mechanics, Non-Relativistic Theory, Pergamon Press, Oxford 1965.

[12] G. Barton, Ann. Phys. 166, 322 (1986).

[13] W. Miller, Symmetry and Separation of Variables, in series Encyclopedia of Mathematics and Its Applications, Addison-Wesley, Reading (MA) 1977.

[14] I.A. Pedrosa, I. Guedes, Int. J. Mod. Phys. B 18 , 1379 (2004).

[15] Problems and Solutions on Quantum Mechanics, Ed. Yung-Kuo Lim, World Sci., Singapore 1998.

[16] M. Abramowitz, I.A. Stegun, Handbook of Mathematical Functions, National Bureau of Standards Applied Mathematics Series 55, 1972.

[17] H. Buchholz, The Confluent Hypergeometric Function, Springer-Verlag, Berlin 1969.

[18] E.T. Whittaker, G.N. Watson, A Course in Modern Analysis, Cambridge University Press, Cambridge (UK) 1996.

[19] P.A.M. Dirac, Proc. R. Soc. A 117, 610 (1928); ibid. 118, 351 (1928).

[20] O. Boyarkin, Advanced Particle Physics: Particles, Fields, and Quantum Electrodynamics Vol. 1, Taylor and Francis, 2011.

[21] V.W. Hughes, T. Kinoshita, Rev. Mod. Phys. 71, 133 (1999). 